DOI: $10.17516 / 1997-1397-2021-14-1-47-56$

УДК 517.95

\title{
Mathematical Model of Fluids Motion in Poroelastic Snow-ice Cover
}

\author{
Margarita A. Tokareva* \\ Alexander A. Papin ${ }^{\dagger}$ \\ Altai State University \\ Barnaul, Russian Federation
}

Received 19.06.2020, received in revised form 16.10.2020, accepted 02.12.2020

\begin{abstract}
The dynamics of a snow-ice cover is considered within the theory of poroelasticity. The snow-ice cover is modeled by a three-phase medium consisting of water, air and ice. The governing equations are the equations of mass conservation for each phase with phase transitions, the equations of conservation of phase momentum in the form of Darcy's law, the equation of conservation of momentum of the whole system, the rheological equation for porosity and the equation of heat balance of snow. In the full formulation the liquid and air pressures are functions of the temperature and the corresponding densities, and the viscosity and compressibility coefficients of ice are functions of the temperature. The problem of two-dimensional nonstationary filtration of water in a thin poroelastic ice plate is considered in the model case. The solution is obtained in quadratures.
\end{abstract}

Keywords: poroelasticity, porosity, snow-ice cover, thin layer.

Citation: M.A. Tokareva, A.A. Papin, Mathematical Model of Fluids Motion in Poroelastic Snow-ice Cover, J. Sib. Fed. Univ. Math. Phys., 2021, 14(1), 47-56. DOI: 10.17516/1997-1397-2021-14-1-47-56.

A large number of studies are devoted to balance models and observations of the behavior of a snow cover [1-3]. These works primarily study snow stocks, flood risks and river flow formation. Models of a melting snow-ice cover based on the approaches of the mechanics of multiphase medium are intended to obtain a more detailed picture of the movement of water and air in melting snow, i.e. determination of the velocity field, concentration, phase pressures, etc. Only a few articles use the "full" models. The problems describing phase transitions and movement of the ice skeleton remain open.

The foundations of the theory of the movement of water and air in the melting snow within the mechanics of multiphase medium are formulated by S. C. Colbeck [4] and his followers [5, 6]. In these works, snow is considered as a multiphase medium, but the variable porosity of ice, its deformation and phase transitions are not taken into account. In this paper, a mathematical model of the movement of water and air in a deformable poroelastic medium is proposed. The model includes the laws of conservation of mass, momentum in the form of Darcy's law for fluid components, the rheological relation for the porosity and the equation for temperature [7]. The obtained system of the equations is a generalization of the well-known system of MusketLeverett equations in the case of variable porosity [8]. We use the concept of effective stress introduced by Terzagi for the porous deformation model when modeling a poroelastic medium. In this approach, the effective stress is defined as the difference between the total stress and the

\footnotetext{
*tma25@mail.ru

†papin@math.asu.ru

(c) Siberian Federal University. All rights reserved
} 
pressure of the liquid phase [9]. This proposition means that the fluid carries part of the load. Fundamental in this approach is the relationship between the deformation of the skeleton of a solid matrix and the processes of fluid flow. Filtration in a thin poroelastic layer is considered in the case of one liquid. The model is based on the theory of multiphase flows in poroelastic medium. Structurally similar mathematical models are used to describe the movement of magma in the earth's crust, the flow of water and oil in a porous medium, the growth of tumors and other filtration processes in poroelastic medium [10-13].

\section{Governing equations}

A system of differential equations describing the movement of air and water in the snowice cover taking into account the phase transitions is considered in the domain $\Omega=(x, z)=$ $=[0, L] \times[0, H]$. The equations of continuity with the variable porosity $\phi$ are

$$
\begin{gathered}
\frac{\partial(1-\phi) \rho_{i}}{\partial t}+\operatorname{div}\left((1-\phi) \rho_{i} \vec{v}_{i}\right)=I_{w i}+I_{a i}, \quad \frac{\partial\left(\rho_{w} s_{w} \phi\right)}{\partial t}+\operatorname{div}\left(\rho_{w} \phi s_{w} \vec{v}_{w}\right)=I_{i w}+I_{a w} \\
\frac{\partial\left(\rho_{a} s_{a} \phi\right)}{\partial t}+\operatorname{div}\left(\rho_{w} \phi s_{a} \vec{v}_{w}\right)=I_{i w}+I_{w a}, \quad s_{a}+s_{w}=1 .
\end{gathered}
$$

Here $\rho_{a}, \rho_{w}, \rho_{i}, \vec{v}_{a}, \vec{v}_{i}, \vec{v}_{w}$ are the true density and phase velocity, respectively ( $a-$ air, $w-$ water, $i$ - ice), $s_{a}, s_{w}$ are the saturations of air and water, respectively, $\phi$ is the porosity, $I_{l m}$ is the intensity of mass transfer from $l$-phase to the $m$-phase per unit volume at time instant, $I_{l m}=-I_{m l}$.

Instead of the equation of dynamics in the theory of two-phase filtration, we use the general Darcy law for liquid phases, taking into account the movement of ice

$$
\begin{gathered}
\phi\left(\vec{v}_{w}-\vec{v}_{i}\right)=-\frac{k(\phi)}{\mu_{w}(\theta)}\left(\nabla p_{w}-\rho_{w} \vec{g}\right), \\
\phi\left(\vec{v}_{a}-\vec{v}_{i}\right)=-\frac{k(\phi)}{\mu_{a}(\theta)}\left(\nabla p_{a}-\rho_{a} \vec{g}\right),
\end{gathered}
$$

where $\theta$ is the temperature of the medium $\left(\theta_{i}=\theta_{w}=\theta\right), p_{w}, p_{a}$ are the water and air pressures, respectively, $k(\phi)$ is the permeability, $\mu_{w}(\theta), \mu_{a}(\theta)$ are the dynamic viscosities of water and air $\vec{g}$ is the gravity acceleration vector. The phase pressures $p_{a}$ and $p_{w}$ differ by the magnitude capillary jump $p_{c}: p_{a}-p_{w}=p_{c}\left(x, s_{w}\right)$.

The Maxwell-type relation between the porosity and the effective pressure $p_{e}$ is [17]

$$
\operatorname{div} \vec{v}_{i}=-\phi\left(\alpha(\theta) p_{e}+\beta(\theta) \frac{d p_{e}}{d t}\right), \quad \frac{d}{d t}=\frac{\partial}{\partial t}+\left(\vec{v}_{i} \cdot \nabla\right),
$$

where $\alpha(\theta), \beta(\theta)$ are the specified environment parameters. The effective pressure $p_{e}$ and the pressures of the liquids $p_{w}, p_{a}$ and solid $p_{i}$ phases are related by the equations

$$
p_{\text {tot }}=\phi p_{f}+(1-\phi) p_{i}, \quad p_{e}=(1-\phi)\left(p_{i}-p_{f}\right), \quad p_{f}=s_{w} p_{w}+s_{a} p_{a} .
$$

The balance equation for the forces of the whole system has the form

$$
\nabla p_{t o t}=\rho_{t o t} \vec{g}+\operatorname{div}\left((1-\phi) \eta(\theta)\left(\frac{\partial \vec{v}_{i}}{\partial \vec{x}}+\left(\frac{\partial \vec{v}_{i}}{\partial \vec{x}}\right)^{*}\right)\right), \quad \rho_{t o t}=\phi \rho_{f}+(1-\phi) \rho_{i}, \quad \rho_{f}=s_{w} \rho_{w}+s_{a} \rho_{a},
$$


where $p_{t o t}$ is the total pressure, $\rho_{\text {tot }}$ is the total density, $\eta(\theta)$ is the viscosity of the porous skeleton, $*$ is the symbol of the transposition operation.

The energy conservation equation is taken as [1]

$$
\begin{gathered}
\left(\rho_{a} c_{a} s_{a} \phi+\rho_{w} c_{w} s_{w} \phi+\rho_{i} c_{i}(1-\phi)\right) \frac{\partial \theta}{\partial t}+\left(\rho_{a} c_{a} s_{a} \phi \vec{v}_{a}+\rho_{w} c_{w} s_{w} \phi \vec{v}_{w}+\rho_{i} c_{i}(1-\phi) \vec{v}_{i}\right) \nabla \theta= \\
=\operatorname{div}\left(\lambda_{t o t}(\phi) \nabla \theta\right)-L_{i} I_{a i}-\nu I_{w i} .
\end{gathered}
$$

Here $c_{i}=$ const $>0, c_{w}=$ const $>0, c_{a}=$ const $>0$ are the heat capacity of ice, water and air at constant volume, respectively; $L_{i}=$ const $>0$ is the specific heat of ice sublimation, $\nu=$ const $>0$ is the specific heat of ice melting; $\lambda_{t o t}$ is the thermal conductivity of the medium as a whole $\left(\lambda_{t o t}=a_{t o t}+b_{t o t} \rho_{t o t}^{2}, a_{t o t}=\right.$ const $>0, b_{t o t}=$ const $\left.>0\right)$. To close the equations, one can take into account the dependence of the densities of liquid phases on pressures and temperatures, or set the densities equal to constant.

Assuming that there is no air in the pores, we shell study the system of differential equations describing the filtration of liquid in the melting ice (the phase densities are constant):

$$
\begin{gathered}
\frac{\partial(1-\phi) \rho_{i}}{\partial t}+\operatorname{div}\left((1-\phi) \rho_{i} \vec{v}_{i}\right)=I_{w i}, \quad \frac{\partial\left(\rho_{w} s_{w} \phi\right)}{\partial t}+\operatorname{div}\left(\rho_{w} \phi s_{w} \vec{v}_{w}\right)=I_{i w}, \\
\phi\left(\vec{v}_{w}-\vec{v}_{i}\right)=-\frac{k(\phi)}{\mu_{w}(\theta)}\left(\nabla p_{w}-\rho_{w} \vec{g}\right), \\
\operatorname{div} \vec{v}_{i}=-\phi\left(\alpha(\theta) p_{e}+\beta(\theta) \frac{d p_{e}}{d t}\right), \quad \frac{d}{d t}=\frac{\partial}{\partial t}+\left(\vec{v}_{i} \cdot \nabla\right), \\
p_{t o t}=\phi p_{w}+(1-\phi) p_{i}, \quad p_{e}=(1-\phi)\left(p_{i}-p_{w}\right), \\
\nabla p_{t o t}=\rho_{t o t} \vec{g}+\operatorname{div}\left((1-\phi) \eta(\theta)\left(\frac{\partial \vec{v}_{i}}{\partial \vec{x}}+\left(\frac{\partial \vec{v}_{i}}{\partial \vec{x}}\right)^{*}\right)\right), \quad \rho_{t o t}=\phi \rho_{w}+(1-\phi) \rho_{i}, \\
\left(\rho_{w} c_{w} \phi+\rho_{i} c_{i}(1-\phi)\right) \frac{\partial \theta}{\partial t}+\left(\rho_{w} c_{w} \phi \vec{v}_{w}+\rho_{i} c_{i}(1-\phi) \vec{v}_{i}\right) \nabla \theta=\operatorname{div}\left(\lambda_{t o t}(\phi) \nabla \theta\right)-\nu I_{w i} .
\end{gathered}
$$

A structurally similar model is used to describe the nonisothermal motion of magma in a poroelastic medium. The system (1)-(6), in the one-dimensional case with constant temperature, the dependence of the density of the liquid phase on the pressure, and in the absence of the phase transitions is studies and the local solvability is proved in [18]. The global solvability is proved in [19] for the same problem when the densities are constant. The numerical analysis of the initial-boundary value problem for system (1)-(6) in the isothermal case is carried out in [20].

\section{Introduction of small parameter}

We shell make equations (1)-(6) dimensionless. Let $\bar{x}, \bar{z}, \bar{t}$ be dimensionless variables defined by the equalities [21]

$$
\bar{x}=\frac{x}{L}, \quad \bar{z}=\frac{z}{H}, \quad \bar{t}=\varepsilon^{l} \tau_{0} t, \quad \varepsilon=\frac{H}{L} \ll 1,
$$

where $[L]=[H]=[\mathrm{m}],\left[\tau_{0}\right]=[1 / \mathrm{s}] ; l$ is a fixed parameter.

The required functions in the dimensionless variables are

$$
p_{w}(t, x, z)=p \bar{p}_{w}(\bar{t}, \bar{x}, \bar{z})=p \bar{p}_{w}\left(\varepsilon^{l} \tau_{0} t, \frac{x}{L}, \frac{z}{H}\right),
$$




$$
\begin{gathered}
p_{i}(t, x, z)=p \bar{p}_{i}(\bar{t}, \bar{x}, \bar{z})=p \bar{p}_{i}\left(\varepsilon^{l} \tau_{0} t, \frac{x}{L}, \frac{z}{H}\right), \\
v_{i}^{j}(t, x, z)=v^{j} \bar{v}_{i}^{j}(\bar{t}, \bar{x}, \bar{z})=v^{j} \bar{v}_{i}^{j}\left(\varepsilon^{l} \tau_{0} t, \frac{x}{L}, \frac{z}{H}\right), \quad j=1,2, \\
v_{w}^{j}(t, x, z)=v^{j} \bar{v}_{w}^{j}(\bar{t}, \bar{x}, \bar{z})=v^{j} \bar{v}_{w}^{j}\left(\varepsilon^{l} \tau_{0} t, \frac{x}{L}, \frac{z}{H}\right), \quad j=1,2,
\end{gathered}
$$

$p_{t o t}(t, x, z)=p \bar{p}_{t o t}(\bar{t}, \bar{x}, \bar{z}), \quad p_{e}(t, x, z)=p \bar{p}_{e}(\bar{t}, \bar{x}, \bar{z}), \quad \theta=\delta \bar{\theta}, \quad I_{w i}=\gamma \bar{I}_{w i}, \quad I_{i w}=\gamma \bar{I}_{i w}$.

Here $\left[v^{i}\right]=[\mathrm{m} / \mathrm{s}],[p]=[\mathrm{Pa}],[\delta]=[\mathrm{K}],[\alpha]=[1 / \mathrm{c}]$, the quantities $p, v^{j}, \delta, \gamma$ are the positive constants. Further, we use the assumption that $k(\phi)=k \phi^{n}, k=$ const, $\eta(\theta)=\eta=$ const $>0$ $[14,17,22]$.

Equations (1)-(6) in scalar form in the dimensionless variables are

$$
\begin{aligned}
& \varepsilon^{l} \tau_{0} \frac{\partial(1-\phi)}{\partial \bar{t}}+\frac{v^{1}}{L} \frac{\partial \bar{v}_{i}^{1}(1-\phi)}{\partial \bar{x}}+\frac{v^{2}}{H} \frac{\partial \bar{v}_{i}^{2}(1-\phi)}{\partial \bar{z}}=\gamma \bar{I}_{w i}, \\
& \varepsilon^{l} \tau_{0} \frac{\partial \phi}{\partial \bar{t}}+\frac{v^{1}}{L} \frac{\partial \bar{v}_{w}^{1} \phi}{\partial \bar{x}}+\frac{v^{2}}{H} \frac{\partial \bar{v}_{w}^{2} \phi}{\partial \bar{z}}=\gamma \bar{I}_{i w}, \\
& \phi\left(v^{1} \bar{v}_{w}^{1}-v^{1} \bar{v}_{i}^{1}\right)=-\frac{k}{\mu} \phi^{n} \frac{p}{L} \frac{\partial \bar{p}_{w}}{\partial \bar{x}}, \\
& \phi\left(v^{2} \bar{v}_{w}^{2}-v^{2} \bar{v}_{i}^{2}\right)=-\frac{k}{\mu} \phi^{n}\left(\frac{p}{H} \frac{\partial \bar{p}_{w}}{\partial \bar{z}}+\rho_{w} g\right), \\
& \frac{v^{1}}{L} \frac{\partial \bar{v}_{i}^{1}}{\partial x}+\frac{v^{2}}{H} \frac{\partial \bar{v}_{i}^{2}}{\partial y}=-\phi\left(\alpha p \bar{p}_{e}-\beta\left(p \varepsilon^{l} \tau_{0} \frac{\partial \bar{p}_{e}}{\partial \bar{t}}+\bar{v}_{i}^{1} \frac{p v^{1}}{L} \frac{\partial \bar{p}_{e}}{\partial \bar{x}}+\bar{v}_{i}^{2} \frac{p v^{2}}{H} \frac{\partial \bar{p}_{e}}{\partial \bar{z}}\right)\right), \\
& 2 \frac{v^{1}}{L^{2}} \frac{\partial}{\partial \bar{x}}\left((1-\phi) \frac{\partial \bar{v}_{i}^{1}}{\partial \bar{x}}\right)+\frac{v^{1}}{H^{2}} \frac{\partial}{\partial \bar{z}}\left((1-\phi) \frac{\partial \bar{v}_{i}^{1}}{\partial \bar{z}}\right)+ \\
& +\frac{v^{2}}{H L} \frac{\partial}{\partial \bar{z}}\left((1-\phi) \frac{\partial \bar{v}_{i}^{2}}{\partial \bar{x}}\right)=\frac{p}{L \eta} \frac{\partial p_{t o t}}{\partial \bar{x}}, \\
& \frac{\left(\rho_{w} \phi+\rho_{i}(1-\phi)\right) g}{\eta} \bar{\rho} \bar{g}+\frac{v^{2}}{L^{2}} \frac{\partial}{\partial \bar{x}}\left((1-\phi) \frac{\partial \bar{v}_{i}^{2}}{\partial \bar{x}}\right)+2 \frac{v^{2}}{H^{2}} \frac{\partial}{\partial \bar{z}}\left((1-\phi) \frac{\partial \bar{v}_{i}^{2}}{\partial \bar{z}}\right)+ \\
& +\frac{v^{1}}{H L} \frac{\partial}{\partial \bar{x}}\left((1-\phi) \frac{\partial \bar{v}_{i}^{1}}{\partial \bar{z}}\right)=\frac{p}{H \eta} \frac{\partial \bar{p}_{t o t}}{\partial \bar{z}}, \\
& \varepsilon^{l} \tau_{0} \delta\left(\rho_{w} c_{w} \phi+\rho_{i} c_{i}(1-\phi)\right) \frac{\partial \bar{\theta}}{\partial \bar{t}}+\frac{v^{1} \delta}{L}\left(\rho_{w} c_{w} \phi \bar{v}_{w}^{1}+\rho_{i} c_{i}(1-\phi) \bar{v}_{i}^{1}\right) \frac{\partial \bar{\theta}}{\partial \bar{x}}+ \\
& +\frac{v^{2} \delta}{H}\left(\rho_{w} c_{w} \phi \bar{v}_{w}^{2}+\rho_{i} c_{i}(1-\phi) \bar{v}_{i}^{2}\right) \frac{\partial \bar{\theta}}{\partial \bar{z}}=\frac{\delta}{L^{2}} \frac{\partial}{\partial \bar{x}}\left(\left(a+b\left(\phi \rho_{w}+(1-\phi) \rho_{i}\right) \frac{\partial \bar{\theta}}{\partial \bar{x}}\right)+\right. \\
& +\frac{\delta}{H^{2}} \frac{\partial}{\partial \bar{z}}\left(\left(a+b\left(\phi \rho_{w}+(1-\phi) \rho_{i}\right)^{2}\right) \frac{\partial \bar{\theta}}{\partial \bar{z}}\right)-\nu \gamma \bar{I}_{w i} .
\end{aligned}
$$

We now choose

$$
v^{1}=\varepsilon^{l} \tau_{0} L, \quad v^{2}=\varepsilon^{l} \tau_{0} H .
$$

Then equations (7)-(12) can be reduced to the form

$$
\begin{gathered}
\frac{\partial(1-\phi)}{\partial \bar{t}}+\operatorname{div}\left((1-\phi) \bar{v}_{i}\right)=\frac{\gamma}{\varepsilon^{l} \tau_{0}} \bar{I}_{w i}, \\
\frac{\partial \phi}{\partial \bar{t}}+\operatorname{div}\left(\phi \bar{v}_{w}\right)=\frac{\gamma}{\varepsilon^{l} \tau_{0}} \bar{I}_{i w},
\end{gathered}
$$




$$
\begin{gathered}
\frac{\varepsilon^{l} \tau_{0} \mu L^{2}}{k p} \phi\left(\bar{v}_{w}^{1}-\bar{v}_{i}^{1}\right)=-\phi^{n} \frac{\partial \bar{p}_{w}}{\partial \bar{x}} \\
\frac{\varepsilon^{l+2} \tau_{0} \mu L^{2}}{k p} \phi\left(\bar{v}_{w}^{2}-\bar{v}_{i}^{2}\right)=-\phi^{n} \frac{\partial \bar{p}_{w}}{\partial \bar{z}}-\frac{\rho_{w} g L \varepsilon}{p} \\
\frac{\varepsilon^{l} \tau_{0}}{\alpha p}\left(\frac{\partial \bar{v}_{i}^{1}}{\partial \bar{x}}+\frac{\partial \bar{v}_{i}^{2}}{\partial \bar{z}}\right)=-\phi \bar{p}_{e}-\frac{\varepsilon^{l} \tau_{0} \beta}{\alpha}\left(\frac{\partial \bar{p}_{e}}{\partial \bar{t}}+\bar{v}_{i}^{1} \frac{\partial \bar{p}_{e}}{\partial \bar{x}}+\bar{v}_{i}^{2} \frac{\partial \bar{p}_{e}}{\partial \bar{z}}\right) \\
2 \varepsilon^{l} \frac{\partial}{\partial \bar{x}}\left((1-\phi) \frac{\partial \bar{v}_{i}^{1}}{\partial \bar{x}}\right)+\varepsilon^{l-2} \frac{\partial}{\partial \bar{z}}\left((1-\phi) \frac{\partial \bar{v}_{i}^{1}}{\partial \bar{z}}\right)+ \\
+\varepsilon^{l} \frac{\partial}{\partial \bar{z}}\left((1-\phi) \frac{\partial \bar{v}_{i}^{2}}{\partial \bar{x}}\right)=\frac{p}{\eta \tau_{0}} \frac{\partial p_{t o t}}{\partial \bar{x}} \\
+\varepsilon^{l} \frac{\partial}{\partial \bar{x}}\left((1-\phi) \frac{\partial \bar{v}_{i}^{1}}{\partial \bar{z}}\right)=\frac{g L \varepsilon \rho_{w}}{\eta \tau_{0}} \phi+\frac{g L \varepsilon \rho_{i}}{\eta \tau_{0}}(1-\phi)+\frac{p}{\eta \tau_{0}} \frac{\partial \bar{p}_{t o t}}{\partial \bar{z}} . \\
\varepsilon^{l}\left(\left(\frac{\rho_{w} c_{w}}{\rho_{i} c_{i}} \phi+(1-\phi)\right) \frac{\partial \bar{\theta}}{\partial \bar{t}}+\left(\frac{\rho_{w} c_{w}}{\rho_{i} c_{i}} \phi \bar{v}_{w}^{1}+(1-\phi) \bar{v}_{i}^{1}\right) \frac{\partial \bar{\theta}}{\partial \bar{x}}+\left(\frac{\rho_{w} c_{w}}{\rho_{i} c_{i}} \phi \bar{v}_{w}^{2}+(1-\phi) \bar{v}_{i}^{2}\right) \frac{\partial \bar{\theta}}{\partial \bar{z}}\right)= \\
=\frac{\partial}{\partial \bar{x}}\left(\left(\frac{a}{L^{2} \tau_{0} \rho_{i} c_{i}}+\frac{b \bar{v}_{i}^{2}}{L^{2} \tau_{0} c_{i}}\left(\left(\frac{\rho_{w}}{\rho_{i}}-1\right) \phi+1\right)^{2}\right) \frac{\partial \bar{\theta}}{\partial \bar{x}}\right)+ \\
+\frac{1}{\varepsilon^{2}} \frac{\partial}{\partial \bar{z}}\left(\left(\frac{a}{L^{2} \tau_{0} \rho_{i} c_{i}}+\frac{b\left(\rho_{w}-\rho_{i}\right)}{L^{2} \tau_{0} \rho_{i} c_{i}} \phi+\frac{b}{L^{2} \tau_{0} c_{i}}\right) \frac{\partial \bar{\theta}}{\partial \bar{z}}\right)-\nu \gamma \bar{I}_{w i} .
\end{gathered}
$$

\section{The passage to the limit and solution by quadratures}

For small $\varepsilon, \varepsilon \rightarrow 0$, the form of equations (13)-(18) depends on the parameter $l$.

We consider the most physical case (for $l=-2$ ) describing a slow flow of the fluid in the poroelastic medium (below, the dashes are omitted):

$$
\begin{gathered}
\frac{\partial(1-\phi)}{\partial t}+\operatorname{div}\left((1-\phi) v_{i}\right)=0 \\
\frac{\partial \phi}{\partial t}+\operatorname{div}\left(\phi v_{w}\right)=0 \\
v_{i}^{1}=v_{w}^{1} \\
\frac{\tau_{0} \mu L^{2}}{k p} \phi\left(v_{w}^{2}-v_{i}^{2}\right)=-\phi^{n} \frac{\partial p_{w}}{\partial z} \\
\frac{\partial v_{i}^{1}}{\partial x}+\frac{\partial v_{i}^{2}}{\partial z}=-\beta p \frac{d\left(p_{t o t}-p_{w}\right)}{d t} \\
\frac{\partial}{\partial z}\left((1-\phi) \frac{\partial v_{i}^{1}}{\partial z}\right)=0 \\
2 \frac{\partial}{\partial z}\left((1-\phi) \frac{\partial v_{i}^{2}}{\partial z}\right)+\frac{\partial}{\partial x}\left((1-\phi) \frac{\partial v_{i}^{1}}{\partial z}\right)=0 \\
-51-
\end{gathered}
$$




$$
\begin{gathered}
\left(\frac{\rho_{w} c_{w}}{\rho_{i} c_{i}} \phi+(1-\phi)\right) \frac{\partial \theta}{\partial t}+\left(\frac{\rho_{w} c_{w}}{\rho_{i} c_{i}} \phi v_{w}^{1}+(1-\phi) v_{i}^{1}\right) \frac{\partial \theta}{\partial x}+\left(\frac{\rho_{w} c_{w}}{\rho_{i} c_{i}} \phi v_{w}^{2}+(1-\phi) v_{i}^{2}\right) \frac{\partial \theta}{\partial z}= \\
=\frac{1}{L^{2} \tau_{0} c_{i}} \frac{\partial}{\partial z}\left(\left(\frac{a}{\rho_{i}}+b\left(\phi\left(\frac{\rho_{w}}{\rho_{i}}-1\right)+1\right)^{2}\right) \frac{\partial \theta}{\partial z}\right) .
\end{gathered}
$$

We outline the scheme of the method of the solution for system (19)-(24). Some of the equations are integrated and as the result of some transformations we arrive at an equation for the function $\phi$.

At the first stage, from equation (19) we find $\operatorname{div} \vec{v}_{i}$ and substitute it into equation (22), then

$$
\frac{\partial G(\phi)}{\partial t}+\vec{v}_{i} \cdot \nabla G(\phi)=\frac{\partial\left(p_{t o t}-p_{w}\right)}{\partial t}+\vec{v}_{i} \cdot \nabla\left(p_{t o t}-p_{w}\right),
$$

where $\frac{d G(\phi)}{d \phi}=\frac{1}{\beta p \phi(1-\phi)}$.

From the last equation we get the equation for $p_{i}$ (if $\phi$ and $p_{w}$ are found):

$$
\frac{\partial U}{\partial t}+v_{i}^{1} \frac{\partial U}{\partial x}+v_{i}^{2} \frac{\partial U}{\partial z}=0,
$$

where $U=G(\phi)+(1-\phi)\left(p_{w}-p_{i}\right)$.

This equation is solved by the method of characteristics, which are defined as the solution of the Cauchy problem

$$
v_{i}^{1}=\frac{d x}{d t}, \quad v_{i}^{2}=\frac{d z}{d t},\left.\quad U\right|_{t=0}=G\left(\phi^{0}\right)+\left(1-\phi^{0}\right)\left(p_{w}^{0}-p_{i}^{0}\right) .
$$

At the second stage, we find a representation for the velocity components. To do this, we twice integrate equation (23) over $z$ and, taking into account (20), we obtain

$$
v_{i}^{1}=v_{w}^{1}=A(x, t) \int_{0}^{z} \frac{1}{1-\phi} d \tau+B(x, t),
$$

where the functions $A(x, t), B(x, t)$ are arbitrary functions of $(x, t)$.

Equation (24) can be written, using (26), as

$$
2 \frac{\partial}{\partial z}\left((1-\phi) \frac{\partial v_{i}^{2}}{\partial z}\right)+\frac{\partial A(x, t)}{\partial x}=0 .
$$

Integrating the last equation over $z$ twice, we obtain a representation for $v_{i}^{2}$ in terms of $\phi$ and arbitrary functions $C(x, t), D(x, t)$ :

$$
v_{i}^{2}=-\frac{1}{2} \frac{\partial A(x, t)}{\partial x} \int_{0}^{z} \frac{\tau}{1-\phi} d \tau+D(x, t) \int_{0}^{z} \frac{1}{1-\phi} d \tau+C(x, t) .
$$

At the third stage, we find representations for the liquid phase. To do this, we summarize equations (19):

$$
\operatorname{div}\left(\phi\left(\vec{v}_{w}-\vec{v}_{i}\right)+\vec{v}_{i}\right)=0 .
$$

Using equations (20) and (21), we have

$$
\operatorname{div} \vec{v}_{i}=\frac{k p}{\tau_{0} \mu L^{2}} \frac{\partial}{\partial z}\left(\phi^{n} \frac{\partial p_{w}}{\partial z}\right) .
$$


Substituting into the last equation the representation for the velocity components of the solid medium, we get the equation for $p_{w}$ :

$$
\begin{gathered}
\frac{\partial}{\partial x}\left(A(x, t) \int_{0}^{z} \frac{1}{1-\phi} d \tau\right)+\frac{\partial B(x, t)}{\partial x}-1 / 2 \frac{\partial A(x, t)}{\partial x} \frac{z}{1-\phi}+ \\
+D(x, t) \frac{1}{1-\phi}=\frac{k p}{\tau_{0} \mu L^{2}} \frac{\partial}{\partial z}\left(\phi^{n} \frac{\partial p_{w}}{\partial z}\right)
\end{gathered}
$$

Integrating this equation twice over $z$, we obtain a representation for $p_{w}$ in terms of $\phi$ and arbitrary functions $E(x, t), F(x, t)$

$$
\begin{gathered}
p_{w}=\frac{\tau_{0} \mu L^{2}}{k p} \int_{0}^{z} \phi^{-n}\left[\int_{0}^{\zeta} \frac{\partial}{\partial x}\left(A(x, t) \int_{0}^{\xi} \frac{1}{1-\phi} d \tau\right) d \xi-\right. \\
\left.-\frac{1}{2} \frac{\partial A(x, t)}{\partial x} \int_{0}^{\zeta} \frac{\tau}{1-\phi} d \tau+\frac{\partial B(x, t)}{\partial x} \zeta+D(x, t) \int_{0}^{\zeta} \frac{1}{1-\phi} d \tau+E(x, t)\right] d \zeta+F(x, t) .
\end{gathered}
$$

Using a representation for $p_{w}$, from (21) we derive the equation for the second component of the velocity $v_{w}^{2}$ in terms of $\phi$

$$
\begin{gathered}
v_{w}^{2}=-\frac{1}{\phi}\left[\int_{0}^{z} \frac{\partial}{\partial x}\left(A(x, t) \int_{0}^{\xi} \frac{1}{1-\phi} d \tau\right) d \xi-\frac{1}{2} \frac{\partial A(x, t)}{\partial x} \int_{0}^{z} \frac{\tau}{1-\phi} d \tau+\right. \\
\left.+\frac{\partial B(x, t)}{\partial x} z+D(x, t) \int_{0}^{z} \frac{1}{1-\phi} d \tau+E(x, t)\right]-\frac{1}{2} \frac{\partial A(x, t)}{\partial x} \int_{0}^{z} \frac{\tau}{1-\phi} d \tau+ \\
+D(x, t) \int_{0}^{z} \frac{1}{1-\phi} d \tau+C(x, t)
\end{gathered}
$$

Thus, the velocity components $\vec{v}_{i}, \vec{v}_{w}$ and the pressure $p_{w}$ are obtained as the functions of $\phi$.

At the last stage, the continuity equation for the solid phase is used to determine the porosity $\phi$ :

$$
\begin{gathered}
\frac{\partial s}{\partial t}+\frac{\partial}{\partial x}\left(s A(x, t) \int_{0}^{z} s^{-1} d \tau+s B(x, t)\right)+ \\
+\frac{\partial}{\partial z}\left(-s \frac{1}{2} \frac{\partial A(x, t)}{\partial x} \int_{0}^{z} \frac{\tau}{s} d \tau+s D(x, t) \int_{0}^{z} s^{-1} d \tau+s C(x, t)\right)=0, \quad s \equiv 1-\phi .
\end{gathered}
$$

Note that the representation for $p_{i}$ is found after obtaining the representation for the components of the velocity of the solid phase and the pressure of the liquid.

Now we consider the following initial-boundary value problem for equations (19)-(24). At $z=0$, the rigid porous skeleton satisfies a plane stress state with the stress tensor $\sigma_{11}=\sigma_{22}=p_{\text {tot }}$, $\sigma_{12}=\sigma_{21}=0$, and the fluid pressure is also set. The velocity of the rigid skeleton is specified at $z=H$, and the phase velocities are equal. The porosity and phase pressures are set at the initial moment of time. Thus, the following initial boundary conditions are considered:

$$
\begin{gathered}
\left.\frac{\partial v_{i}^{2}}{\partial z}\right|_{z=0}=0,\left.\quad v_{i}^{2}\right|_{z=H}=C=\text { const, } \\
\left.v_{i}^{1}\right|_{z=H}=B=\text { const },\left.\quad \frac{\partial v_{i}^{1}}{\partial z}\right|_{z=0}=0, \\
\left.\phi\right|_{t=0}=\phi^{0}(x, t),\left.\quad \frac{\partial p_{w}}{\partial z}\right|_{z=H}=0, \\
\left.p_{w}\right|_{t=0}=p_{w}^{0}(x, z),\left.\quad p_{i}\right|_{t=0}=p_{i}^{0}(x, z),\left.\quad p_{w}\right|_{z=0}=p_{0}(x, t), \\
-53-
\end{gathered}
$$


where the constants $B$ and $C$ are given. This allow us to uniquely define the following functions:

$$
\begin{gathered}
A(x, t)=0, \quad B(x, t)=B=\mathrm{const}, \quad C(x, t)=C=\mathrm{const}, \\
D(x, t)=0, \quad E(x, t)=0, \quad F(x, t)=p_{0}(x, t), \\
v_{i}^{1}=v_{w}^{1}=B, \quad v_{i}^{2}=v_{w}^{2}=C, \quad p_{w}=p_{0}(x, t),
\end{gathered}
$$

and the equation for $s$ can be rewritten as

$$
\frac{\partial s}{\partial t}+B \frac{\partial s}{\partial x}+C \frac{\partial s}{\partial z}=0 .
$$

The solution of this equation is

$$
\phi=\phi^{0}(x-B t, z-C t) .
$$

The solution for $p_{i}$ is

$$
p_{i}=p_{0}(x, t)-p_{w}^{0}(x-B t, z-C t)+p_{i}^{0}(x-B t, z-C t) .
$$

The equation for $\theta$ takes the form

$$
\begin{aligned}
& \left(\frac{\rho_{w} c_{w}}{\rho_{i} c_{i}} \phi^{0}(x-B t, z-C t)+\left(1-\phi^{0}(x-B t, z-C t)\right)\right)\left(\frac{\partial \theta}{\partial t}+B \frac{\partial \theta}{\partial x}+C \frac{\partial \theta}{\partial z}\right)= \\
= & \frac{1}{L^{2} \tau_{0} c_{i}} \frac{\partial}{\partial z}\left(\left(\frac{a}{\rho_{i}}+b \rho_{i}\left(\phi^{0}(x-B t, z-C t) \frac{\rho_{w}}{\rho_{i}}+1-\phi^{0}(x-B t, z-C t)^{2}\right)\right) \frac{\partial \theta}{\partial z}\right)
\end{aligned}
$$

and is supplemented by the following initial-boundary conditions

$$
\left.\theta\right|_{t=0}=\theta^{0}(x, z),\left.\quad \frac{\partial \theta}{\partial z}\right|_{z=0, z=H}=0,\left.\quad \frac{\partial \theta}{\partial x}\right|_{x=0, x=L}=0 .
$$

The equation for $\theta$ is linear uniformly parabolic. The maximum principle for the function $\theta$ is valid, and theorem of existences is also established for this problem (q.v. [23], pp. 29, 364).

Thus, the solution of problem (19)-(25), (27), (28) is found.

\section{Conclusion}

A new model of the dynamics of the snow-ice cover, taking into account the deformation of the porous iced skeleton, is considered in this work. The model is a generalization of the classical Musket-Leverett two-phase filtration model and it is applicable for a wide class of problems of multiphase flows in a poroelastic media. A feature of the model is the variable porosity. The problem of the justification of this model is open. There are also no results on the construction of simple solutions. On the other hand, the study of such a general problem is important for the analysis of complicated processes of salt-mass transfer in a melting snow. For example, the speed of pollution in spring during melting of the snow cannot be described without knowing the field of velocities and water saturation. Using a sophisticated model allows to get a complete picture of the dynamics of the process. The important point is the correct choice of initial data, which guarantees the existence of a solution. Only examples of single-phase numerical solutions are known without phase transitions and temperature effects [14,22]. In this article, we consider a simplified model based on the selection of a small parameter, which is a common technique in 
the study of complex problems. Solutions in quadratures are constructed. In the future, we plan to study the justification for the formulated problem and its applications in polar mechanics.

The work was carried out under the project MK-204.2020.1 "Initial-boundary value problems for the equations of fluid motion in poroelastic media and their applications in the dynamics of snow and ice cover" with the support of a grant from the President of the Russian Federation.

\section{References}

[1] L.S.Kuchment, P.Romanov, A.N.Gelfan, V.N.Demidov, Use of satellite-derived data for characterization of snow cover and simulation of snowmelt runoff through a distributed physically based model of runoff generation, Hydrol. Earth Syst. Sci., 14(2010), 339-350.

DOI: $10.5194 /$ hess-14-339-2010

[2] A.N.Gelfan, J.W.Pomeroy, L.S. Kuchment, Modeling Forest Cover Influences on Snow Accumulation, Sublimation, and Melt, Journal of Hydrometeorology, 5(2004), 785-803.

[3] M.Sturm, J.Holmgren, G.Liston, A seasonal snow cover classification-system for local to global applications, Journal of Climate, 8(1995), no. 5, 1261-1283.

DOI: $10.1175 / 1520-0442(1995) 008<1261$ :ASSCCS $>2.0$. CO $; 2$

[4] S.C.Colbeck, A theory of water percolation in snow, Journal Glaciol, 11(63)(1972), 369-385.

[5] J.M.N.T.Gray, Water movement in wet snow, Philosophical Transactions: Mathematical, Physical and Engineering Sciences, 354 (1707)(1996), 465-500.

[6] S.Sellers, Theory of water transport in melting snow with a moving surface, Cold Regions Science and Technology, 31(2000), no. 1, 47-57. DOI: 10.1016/S0165-232X(00)00006-9

[7] R.I.Nigmatullin, Dynamics of multiphase media, Vol. 1, Moscow, Nauka, 1987 (in Russian).

[8] S.N.Antontsev, A.V.Kazhikhov, V.N.Monakhov, Boundary-Value Problems of the Mechanics of Inhomogeneous Fluids, Novosibirsk, Nauka, 1983 (in Russian).

[9] K.Terzaghi, Theoretical Soil Mechanics, New York, Jhon Wiley, 1943.

[10] V.V.Vedernikov, V.N.Nikolaevsii, Mechanics equations for porous medium saturated by a two-phase liquid, Izvestiya Akademii Nauk SSSR, Mekhanika Zhidkosti i Gaza, 5(1978), $769-773$.

[11] Y.Geng, L.Zhang, Bifurcations of traveling wave solutions for the magma equation, Applied Mathematics and computation, 217(2010), 1741-1748. DOI: 10.1016/j.amc.2009.11.035

[12] G.Simpson, M.Spiegelman, M.I.Weinstein, Degenerate dispersive equations arising in the study of magma dynamics, Nonlinearity, 20(2007), 21-49.

[13] A.Friedman, Cancer as Multifaceted Disease, Math. Model. Nat. Phenom., 7(2012).

[14] J.A.D.Connolly, Y.Y.Podladchikov, Temperature-dependent viscoelastic compaction and compartmentalization in sedimentary basins, Tectonophysics, 324(2000), 131-135.

[15] J.Bear, Dynamics of Fluids in Porous Media, Elsevier, New York, 1972. 
[16] A.A.Papin, Y.Y.Podladchikov, Isothermal motion of two immiscible fluids in poroelastic medium, Izvestiya Altai State University, 1/2(85)(2015), 131-135 (in Russian).

DOI: $10.14258 / \operatorname{izvasu}(2015) 1.2-24$

[17] A.Fowler, Mathematical Geoscience, Springer-Verlag, London Limited, 2011.

[18] A.A.Papin, M.A.Tokareva, On Local Solvability of the System of the Equations of One Dimensional Motion of Magma, Journal of Siberian Federal University. Mathematics and Physics, 10(2017), no. 3, 362-371. DOI: 10.17516/1997-1397-2017-10-3-362-371

[19] M.A.Tokareva, A.A.Papin, Global solvability of a system of equations of one-dimensional motion of a viscous fluid in a deformable viscous porous medium, Journal of Applied and Industrial Mathematics, 13(2019), no. 2, 350-362. DOI: 10.33048/sibjim.2019.22.208

[20] M.N.Koleva, L.G.Vulkov, Numerical analysis of one dimensional motion of magma without mass forces, Journal of Computational and Applied Mathematics, 366(2020), 112338. DOI: 10.1016/j.cam.2019.07.003

[21] J.Escher, M.Hillairet, P.Laurencot, C.Walker, Thin film equations with soluble surfactant and gravity: modeling and stability of steady states, Mathematische Nachrichten, 285(2012), $210-222$.

[22] C.Morency et al., A numerical model for coupled fluid flow and matrix deformation with applications to disequilibrium compaction and delta stability, Journal of Geophysical Research: Solid Earth, 112(2007), no. B10.

[23] O.A.Ladyzhenskaya, V.A.Solonnikov, N.N.Ural'tseva, Linear and Quasilinear Equations of Parabolic Type, Moscow, Nauka, 1967 (in Russian).

\title{
Математическая модель движения жидкостей в пороупругом снежно-ледовом покрове
}

Маргарита А. Токарева Александр А. Папин Алтайский государственный университет Барнаул, Российская Федерация

\begin{abstract}
Аннотация. Задача динамики снежно-ледового покрова рассматривается в рамках теории пороупругости. Снежно-ледовый покров рассматривается как трехфазная среда, состоящая из воды, воздуха и льда. В основу математической модели положены уравнения сохранения массы для каждой из фаз с учетом фазовых переходов, уравнения сохранения импульсов фаз в форме законов Дарси, уравнение сохранения импульса системы в целом, реологического уравнения для пористости и уравнение теплового баланса снега. В полной постановке учтена зависимость давлений жидкости и воздуха от температуры и соответствующих плотностей, а также зависимость коэффициентов вязкости и сжимаемости льда от температуры. В модельном случае рассматривается фильтрация воды в тонкой пороупругой ледовой пластине. Получены решения в квадратурах.
\end{abstract}

Ключевые слова: пороупругость, пористость, снежно-ледовый покров, тонкий слой. 